\title{
Classification of Power Quality Disturbances using Mahalanobis Distance Classifier and Stockwell Transformation
}

\author{
Md. Jashim Uddin Bhuiyan, and M. R. Alam
}

\begin{abstract}
Detection and classification of PQ (Power Quality) disturbances in distribution/transmission systems are very important for protection of electricity network. Most of the disturbances of power network are non-stationary and momentary in nature, hence it requires advanced tools and techniques for the analysis and classification of $P Q$ disturbances. This paper presents the detection and classification of $P Q$ events or disturbances employing Stockwell-Transformation, known as S-Transformation, and Mahalanobis Distance (MD) based approach. The proposed method exploits only four features extracted through S-transformation of the voltage signal; then, using these four features, classification is conducted by MD based classifier. In this work, classification of several $P Q$ disturbances, such as, voltage sags, swells, harmonics, notch, flicker, transient oscillation, momentary interruption, etc., are considered. The simulation results demonstrate that the proposed method is very effective and accurate in detecting and classifying $P Q$ events. Validation of the proposed approach has also been conducted using real signal recorded in IEEE 1159.2 database. Moreover, comparative classification performance of MD based classifier with MED (minimum Euclidean distance) and LVQ (learning vector quantization) reveals the superiority of the proposed approach.
\end{abstract}

Keywords-power quality events; classification; feature extraction; S-transform; Mahalanobis distance based classifier.

\section{INTRODUCTION}

The quality of electric power due to the disturbances in power system has become a foremost issue among the electric power suppliers and customers. For refining the PQ (power quality), continuous monitoring of power is needed which is being delivered at customer's sites. Therefore, detection of PQ disturbances, and proper classification of Power Quality Disturbances is most desirable. The equipment, which are being

\section{Md. Jashim Uddin Bhuiyan}

American International University-Bangladesh (AIUB)

Banani, Dhaka 1213, Bangladesh

bhuiyan08103@gmail.com

\section{Dr. Mollah Rezaul Alam}

Assistant Professor

American International University-Bangladesh (AIUB)

Banani, Dhaka 1213, Bangladesh

mra015@aiub.edu used in electrical utilities, are sensitive to Power Quality. Moreover, this equipment contains power electronic components, which are also sensitive to power disturbances. Hence, any type of disturbance occurs in the utility side, it affects the voltage, current or frequency of the power signal which in turn affects the customer's side and the issue is known as power quality problem. Therefore, electrical utilities and customers both are aware of the power quality disturbances. Disturbances and/or faults in power transmission or distribution lines are normally considered as the primary reason to occur PQ events, such as, momentary interruption, flicker, notch, voltage sag/swell with and without harmonics, harmonic distortion, and transients, etc. Faults in an electricity network may create voltage sag or interruption, whereas disconnection of large load or capacitor bank energization may result in voltage swell [1]. In contrast, harmonic distortion and notching in the voltage and current may occur due to the usage of nonlinear power electronic loads, such as, electronic inverters or rectifiers. Flickers are usually found due to the use of arc furnaces. Transients are frequently found in electricity networks and the influential factors of transients are transformer energization, heavy load or capacitor switching, etc. The PQ events can have adverse effect on the electrical equipment, e.g., malfunctions, short lifetime, and failure of equipment may occur. Therefore, at the first stage, detection and accurate classification of PQ events are required, so that appropriate mitigation and fault clearance technique can be deployed. Hence, in this paper, identification (detection and classification) of 10 PQ events (including the normal voltage waveforms), which are frequently found in power system, is conducted employing $\mathrm{S}$ transformation (Stockwell Transformation and Mahalanobis Distance (MD) based classifier.

\section{PQ Events And Their Mathematical REPRESENTATION}

Disturbances or faults in electricity network may lead to malfunction or even failures of different sensitive equipment. To quantify and characterize the severity of disturbances or faults, firstly, different types of PQ events, such as, sags, swells, harmonics and transients, are needed to be detected instantly and classified accurately. In this Section, the definition and the causes of some important PQ disturbances are discussed along with their mathematical expressions in Table I.

1) Voltage Sag: Voltage sag indicates the reduction of rms voltage or current to a level of 0.1 to 0.9 pu for a typical duration 
of 0.5 cycle to 1 minute. Usually, voltage sags are initiated by rapid increases in loads, such as, electrical short circuits or faults, starting of induction motors, or they may be initiated by sudden rises in source impedance, typically occurred by a loose connection.

2) Voltage Swell: Voltage swell is found due to the increase in rms voltage or current resulting in 1.1 to $1.8 \mathrm{pu}$ for a typical duration of 0.5 cycle to 1 minute. Normally, voltage swells are initiated by a rapid reduction of load in a circuit which is poorly regulated, although they may also be triggered by a broken or loose neutral wire connection.

3) Voltage Transient: According to IEEE 1159.2 standard, voltage transient is defined by the fluctuation of voltage which lies in the range of 0-4 pu for a duration of 0.3-50 ms [1].

4) Harmonics: Harmonics of voltage or current signals are sinusoidal in nature and they contain the frequencies which are integer multiples of the fundamental frequency of the designed system. An ideal (pure) sinusoidal waveform is produced by an ideal AC alternator, which is designed with stator and field winding operating in a uniformly distributed magnetic field. However, in practice, uniformly distributed magnetic field is usually not found; hence, distortion of voltage waveforms are created and consequently, the instantaneous voltage waveforms deviate from the pure (ideal) sinusoidal wave. Since there is a deviation from pure sinusoidal wave and the deviation occurs while maintaining the periodicity of sinusoidal wave pattern, therefore, by definition, the voltage distortion yields harmonics [2].

5) Interruption: Interruption occurs when the voltage or load current reduces and reaches to a level of $0.1 \mathrm{pu}$ or less for a duration of less than $1 \mathrm{~min}$. Typically, duration of interruption are measured and recorded as an important parameter since the voltage level remains less than $10 \%$ of nominal voltage during interruption.

6) Flicker: They are cyclical variations in the voltage rms values or a series of random voltage changes, whose magnitude does not normally exceed voltage ranges of 0.9 p.u. to 1.1 p.u. A common phenomenon of voltage fluctuations is the voltage flicker. Loads, which can exhibit continuous, rapid variations in the load current magnitude, can cause voltage fluctuations or flickers. The present industry practice is to characterize the severity of a voltage flicker with respect to the sensitivity of the human visual perception. Typically, magnitudes as low as $0.5 \%$ can result in a perceptible lamp flicker. Arc furnace and welders are the most common causes of voltage fluctuations in utility transmission and distribution systems. Other sources of voltage fluctuation include lumber mills, draglines, and rock crushing machines. Voltage fluctuations can be considered as repetitive random voltage sags and swells. Therefore, voltage fluctuations have propagation characteristics similar to those of sags. They can be assessed using steady-state power system models. A strong supply system can greatly reduce severity of voltage fluctuation. Voltage fluctuations can cause incandescent and fluorescent lights to blink rapidly. It can also cause sensitive equipment to malfunction. Static VAR system can mitigate the flicker effects [3]. As the annoyance created by flicker is a function of both the intensity of perception and the duration of exposure, according to IEC the severity of the disturbance is described by two parameters: the short-term severity $\left(\mathrm{P}_{\mathrm{st}}\right)$ and the long term severity $\left(\mathrm{P}_{\mathrm{lt}}\right)$. Pst is measured over a period of ten minutes whereas the long term severity $\left(\mathrm{P}_{\mathrm{lt}}\right)$ is calculated from a sequence of $12 \mathrm{P}_{\mathrm{st}}$ values over a two hour interval.

7) Notching: It is a periodic voltage disturbance caused by the normal operation of power electronics devices when current is commutated from one phase to another. Notching can be characterized through the harmonic spectrum of the affected voltage. Although notching is a special case of voltage harmonics, it is generally treated as an independent disturbance. The frequency components associated with notching can be quite high and may not be readily measured with equipment normally used for harmonic analysis. It is defined as unwanted electrical signals with broadband spectral content lower than $200 \mathrm{kHz}$, superimposed upon the power system voltage or current in phase conductors, or found in neutral conductors or signal lines. Power electronic devices can cause noise in power systems, control circuits, and arcing equipment. Improper grounding that fails to conduct noise away from the power system often exacerbates noise problems. Basically, noise consists of any unwanted distortion of the power signal that cannot be classified as harmonic distortion or a transient. Noise disturbs electronic devices such as microcomputers and programmable controllers [4].

Table I exhibits the mathematical representation of $10 \mathrm{PQ}$ events, which are voltage sag, swell, harmonic, sag with harmonic, swell with harmonic, notch, flicker, interruption, transients along with normal pure sinusoid waveform. In Table $I, t_{1}$ is the PQ event inception time and $t_{2}$ is the event ending time, $\mu(t)$ is the unit step function represented by (1).

$$
\mu(t)= \begin{cases}0, & t \leq 0 \\ 1, & t>0\end{cases}
$$

TABLE I. ANALYTICAL EXPRESSIONS OF 10 PQ EVENTS

\begin{tabular}{|c|c|c|}
\hline PQ Events & Equations & Parameters \\
\hline Normal & $v(t)=\sin \left(\omega_{0} t\right)$ & $f_{0}=50 \mathrm{~Hz}$ \\
\hline Sag & $v(t)=\left(1-\alpha\left(\mu\left(t-t_{1}\right)-\mu\left(t-t_{2}\right)\right)\right) \sin \left(\omega_{0} t\right)$ & $\alpha=0.1 \sim 0.9$ \\
\hline Swell & $v(t)=\left(1+\alpha\left(\mu\left(t-t_{1}\right)-\mu\left(t-t_{2}\right)\right)\right) \sin \left(\omega_{0} t\right)$ & $\alpha=0.1 \sim 0.8$ \\
\hline Harmonics & $\begin{array}{l}v(t)=\sin \left(\omega_{0} t\right)+\alpha_{3} \sin \left(3 \omega_{0} t\right)+ \\
\alpha_{5} \sin \left(5 \omega_{0} t\right)\end{array}$ & $\begin{array}{l}\alpha_{3}=\alpha_{5},= \\
0.05 \sim 0.15\end{array}$ \\
\hline $\begin{array}{l}\text { Sag with } \\
\text { harmonics }\end{array}$ & $\begin{array}{l}v(t)=\left(1-\alpha\left(\mu\left(t-t_{1}\right)-\mu\left(t-t_{2}\right)\right)\right) \\
\left(\sin \left(\omega_{0} t\right)+\alpha_{3} \sin \left(3 \omega_{0} t\right)+\alpha_{5} \sin \left(5 \omega_{0} t\right)\right)\end{array}$ & $\begin{array}{c}\alpha=0.1 \sim 0.9 \\
\alpha_{3}=\alpha_{5},= \\
0.05 \sim 0.15\end{array}$ \\
\hline $\begin{array}{l}\text { Swell with } \\
\text { harmonics }\end{array}$ & $\begin{array}{l}v(t)=\left(1+\alpha\left(\mu\left(t-t_{1}\right)-\mu\left(t-t_{2}\right)\right)\right) \\
\left(\sin \left(\omega_{0} t\right)+\alpha_{3} \sin \left(3 \omega_{0} t\right)+\alpha_{5} \sin \left(5 \omega_{0} t\right)\right)\end{array}$ & $\begin{array}{c}\alpha=0.1 \sim 0.8 \\
\alpha_{3}=\alpha_{5},= \\
0.05 \sim 0.15\end{array}$ \\
\hline Interruption & $v(t)=\left(1-\alpha\left(\mu\left(t-t_{1}\right)-\mu\left(t-t_{2}\right)\right)\right) \sin \left(\omega_{0} t\right)$ & $\alpha=0.9 \sim 1$ \\
\hline Flicker & $v(t)=\sin \left(\omega_{0} t\right)\left(1+\beta \sin \left(\gamma \omega_{0} t\right)\right)$ & $\begin{aligned} \beta= & 0.1 \sim 0.2, \gamma= \\
& 0.1 \sim 0.2\end{aligned}$ \\
\hline Notch & $v(t)=\sin \left(\omega_{0} t\right)+0.05 *\left(\sin \left(\omega_{0} t\right) *\right.$ noise & $\begin{array}{l}\text { noise }=\text { white } \\
\text { Gaussian } 25 \\
\text { dB }\end{array}$ \\
\hline Transient & $\begin{array}{l}v(t)=\sin \left(\omega_{0} t\right)+ \\
\alpha * e^{-c\left(t-t_{1}\right)} * \sin \left(\beta \omega_{0} t\right) *\left(\mu\left(t-t_{1}\right)-\mu\left(t-t_{2}\right)\right)\end{array}$ & $\begin{aligned} \alpha & =0.1 \sim 0.8, \beta \\
& =0.1 \sim 0.5\end{aligned}$ \\
\hline
\end{tabular}




\section{PROPOSED APPROACH FOR DETECTION AND CLASSIFICATION OF PQ EVENTS}

Wavelet Transform (WT) exhibits its notable capabilities for detection and localization of the disturbances [5]-[6]. However, its capabilities are often significantly degraded in real practice under noisy environment. On the other hand, STransform (ST), introduced by R. G. Stockwell in 1996, has the ability to detect the disturbance correctly in the presence of noise. This ability of S-Transform attracts the researchers for the detection and classification of PQ disturbances. The ST is useful in detecting and extracting disturbance features of various types of electric power quality disturbances. Basically, ST is an invertible time-frequency spectral localization technique, which combines elements of WT and STFT (Short Time Fourier Transform). It can also be represented as a continuous wavelet transform with a phase correction. It produces a constant relative bandwidth analysis like wavelets, although maintains a direct link with Fourier spectrum. The ST has an advantage in that it provides multi-resolution analysis while retaining the absolute phase of each frequency. This has led to its application for detection and interpretation of nonstationary signals. Further, the ST provides frequency contours which clearly localize the signals at a higher noise level. The major advantage over WT of ST is to avoid the requirement of testing various families of wavelets to identify the best one for a better classification. Furthermore, ST can classify four types of PQ disturbances by visualizing the time-frequency contour. The output of the ST is an $\mathrm{M} \times \mathrm{N}$ matrix, whose rows pertain to frequency and columns indicate time. Each column thus represents the "local spectrum" for that point in time. To construct an effective classifier, it is essential to choose a suitable feature vector that can indicate and recognize the main characteristics of signal. For this purpose, the statistical features based on ST, which indicates and recognizes the main characteristics of signal without losing its distinguishing characteristics, is extracted in the proposed approach discussed in Sections III-C and III-D.

\section{A. Discrete S-Transformation}

Let $x(t)$ denote a continuous time signal, which is sampled with a sampling interval $T_{s}$; the sampling process yields a discrete time series $x\left(k T_{s}\right), k=0,1,2, \ldots \ldots, \mathrm{N}-1$.

The DFT (Discrete Fourier Transform) of the signal $x\left(k T_{s}\right)$ is given by

$$
X\left[\frac{n}{N T_{S}}\right]=\sum_{k=0}^{N-1} x\left(k T_{S}\right) e^{-2 \pi i n k / N}
$$

Where $n=0,1,2, \ldots, N-1$ and the IDFT (Inverse Discrete Fourier Transform) is

$$
x\left(k T_{S}\right)=\frac{1}{N} \sum_{n=0}^{N-1} X\left[\frac{n}{N T_{S}}\right] e^{2 \pi i n k / N}
$$

In discrete-time domain, if the vectors defined by $x\left(k T_{s}\right)$ are observed, it is revealed that $\mathrm{S}$-Transform is merely the projection of those vectors onto a spanning set of vectors [12]. Now, the S-Transform of $x\left(k T_{s}\right)$ is given by [12]

$$
S\left(\frac{n}{N T_{S}}, j T_{S}\right)=\sum_{m=0}^{N-1} X\left[\frac{m+n}{N T_{S}}\right] G(n, m) e^{j 2 \pi i m k / N}
$$

where $G(n, m)=e^{-\left(2 \pi^{2} m^{2} / n^{2}\right)}$ is a Gaussian function and $j, m, n$ $=0,1,2,3, \ldots \ldots, N-1$. By adopting the following steps, discrete $\mathrm{S}$-Transformation of a signal are computed.

- Step 1: DFT of the discrete-time signal $x\left(k T_{s}\right)$ is conducted to obtain $X\left[m / N T_{S}\right]$

- $\quad$ Step 2: For the target frequency $\left(n / N T_{s}\right)$, the Gaussian function $G(n, m)$ is computed.

- $\quad$ Step 3: By employing one pointer addition, $X\left[m / N T_{S}\right]$ is shifted to $X\left[(m+n) / N T_{S}\right]$ for the target frequency $\left(n / N T_{s}\right)$.

- $\quad$ Step 4: $G(n, m)$ is multiplied by $X\left[(m+n) / N T_{S}\right]$ to acquire $B\left[n / N T_{S}, m / N T_{S}\right]$.

- Step 5: IDFT of $B\left[n / N T_{S}, m / N T_{S}\right]$ yields $S\left(n / N T_{S}, j T_{S}\right)$ corresponding to the $j$ row and frequency $\left(n / N T_{s}\right)$.

- $\quad$ Step 6: Steps 3, 4, and 5 are repeated until all the rows of $S\left(n / N T_{S}, j T_{S}\right)$ corresponding to all discrete frequencies are obtained.

From (4) it is evident that output of S-transform is an $\mathrm{N} \times \mathrm{M}$ complex matrix (S-matrix) where columns are associated with time and rows are associated with frequency.

\section{B. Mahalanobis Distance}

Mahalanobis Distance (MD), which is introduced by P.C. Mahalanobis in 1936, is a unique method for distance measurement. It is based on correlation between variables or samples which are used to analyze different patterns of a system. Mathematically, it can be given by

$$
M D(x)=\sqrt{(x-\mu)^{T} S^{-1}(x-\mu)}
$$

where $x=\left(x_{1}, x_{2}, \ldots, x_{N}\right)^{T}$, is the multivariate vector, $\mu=\left(\mu_{1}, \mu_{2}, \ldots . \mu_{N}\right)^{\mathrm{T}}$ is the mean/average of the group of samples or values from which MD is measured and $S=$ covariance matrix of the group of samples [7].

\section{Feature Extraction}

S-transformation gives large time-resolution at higher frequency and large frequency resolution at low frequency; due to these properties of S-transform, it can be very effective for nonstationary power signal. In this work, the sampling frequency of $3.2 \mathrm{kHz}$ is kept during the simulation of 10 types of PQ events as well as the features extraction from $\mathrm{S}$-Transform.

From S-matrix magnitude and frequency can be extracted and to support this statement, Fig. 1 is shown as an example, which illustrates a sag signal and its S-Transformed features. In Fig. 1(a), the dash line represents the locus of a curve which is 
obtained by taking the maximum value of the elements residing in each column of S-matrix corresponding to the respective time. Fig. 1(b) illustrates the frequency contour of S-matrix. Fig. 1(c) exhibits the maximum magnitude of the complex variables present in each row and it indicates the maximum amplitude present in each frequency components of the signal.

(a)

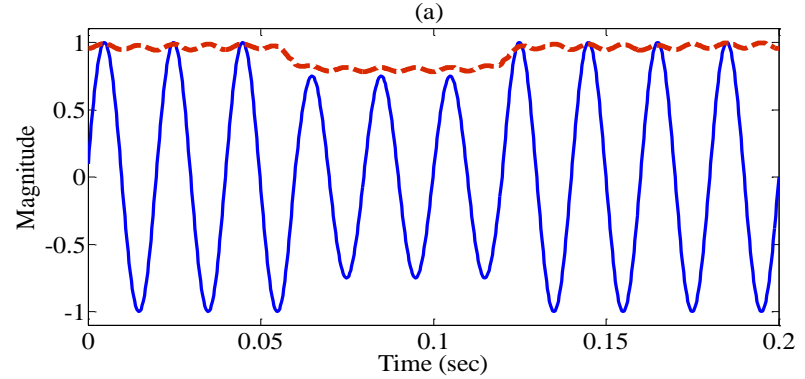

(b)

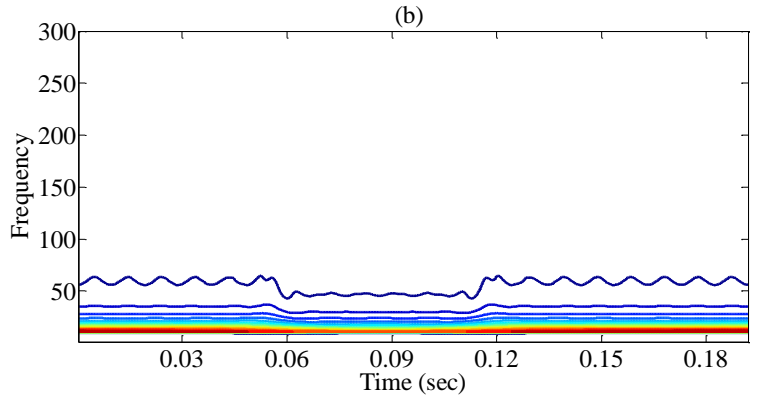

(c)

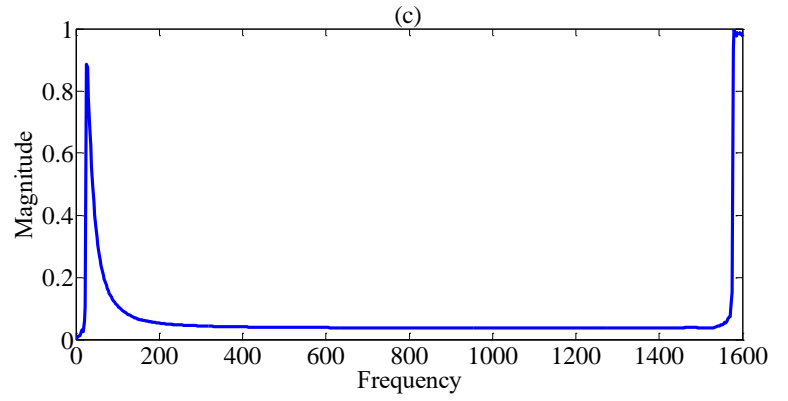

Fig. 1. Voltage sag signal and its S-Transformed feature waveforms.

Feature extraction is a crucial task for classification and in this paper, this task (feature extraction) is conducted by taking standard deviation (SD) and energy of the S-Transformed signal in the following way:

- Feature 1 (F1): SD of the set of data extracted from the elements corresponding to maximum absolute value of each column of S-matrix.

- Feature 2 (F2): Energy of the set of data extracted from the elements corresponding to maximum absolute value of each column of S-matrix.

- Feature 3 (F3): SD of the set of data extracted from the elements corresponding to maximum absolute value of each row of S-matrix.

- Feature 4 (F4): Energy of the set of data extracted from the elements corresponding to maximum absolute value of each row of S-matrix.

\section{Proposed Algorithm}

The proposed approach requires S-transformation on the voltage signal for feature extraction; see the detailed feature extraction procedure in Section III-C. Then, minimum distance based MD classification rule is applied to classify the PQ events. This paper deals with the classification of 10 classes or groups of PQ events; the groups are: G1 (pure normal signal), G2 (sag signal), G3 (swell signal), G4 (harmonic), G5 (sag with harmonic), G6 (swell with harmonic), G7 (notch signal), G8 (flicker signal), G9 (transient), and G10 (momentary interruption). Firstly, several PQ events corresponding to 10 groups are generated by using the equations presented in Table I. Then, STransformation is applied on the 10 groups of signal to extract four features for each event. Thereafter, a reference group of feature matrix labeled with group number is constructed.

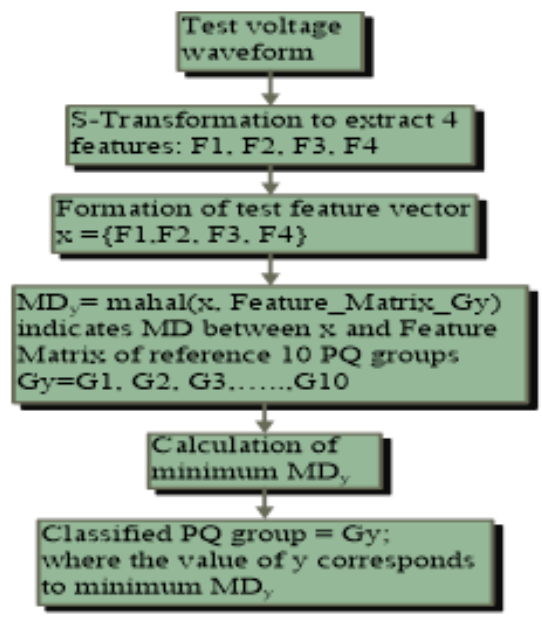

Fig. 2. Flowchart of the proposed approach.

Lastly, classification task is conducted by following the proposed algorithm shown in Fig. 2. As shown in the flowchart of Fig. 2, firstly, a single phase instantaneous voltage waveform (in this case test event) is passed through the S-Transformation followed by 4 features (F1, F2, F3, F4) extraction. Then, classification task is carried out on the principle of least Mahalanobis distance (MD) between the feature vector of test event and the feature matrix of reference groups. For example, if the MD between features of test event and the feature matrix of "reference sag group" is minimum, then the test event experiences sag.

\section{RESULTS AND DISCUSSIONS}

This Section presents the test results associated with the classification of 10 groups of PQ events. To this end, firstly, a total of $1800 \mathrm{PQ}$ events are generated by using the equations shown in Table I. Each group or class contains 180 events, among which 80 events are used to form the reference group feature matrix (see Section III-D) and the remaining 100 events are used for testing. Therefore, a total of 800 events are used for building the reference group-wise feature matrix and 1000 events are used for testing or evaluating the performance of the proposed approach. Note that different PQ events belonging to the same group are generated by varying several parameters, e.g., for the sag case, $\alpha$ is varied from 0.1 to 0.9 (see Table I) 
with a step-size of 0.01 along with the variation of $t_{1}$ (inception time) and $\mathrm{t}_{2}$ (ending time).

Classification results of the S-transform and MD based method are shown in Table II, where the diagonal elements (written in bold) represent the number of successfully classified events and off-diagonal elements indicate the number of misclassified PQ events. The overall accuracy is obtained from the ratio of the number of successfully classified PQ events to the total number of PQ events. Table II highlights the successful classification of all groups of PQ events with overall accuracy of $95.4 \%$. However, to test the robustness of the proposed approach, noise is added at different level which ranges from SNR (Signal-to-noise-ratio) of $20 \mathrm{~dB}$ to $40 \mathrm{~dB}$. The test results show overall accuracy of above $90 \%$ under noisy condition, see Table III.

TABLE II. CLASSIFICATION OF PQ EVENTS USING MD CLASSIFIER

\begin{tabular}{|c|c|c|l|l|l|l|l|c|c|c|}
\hline & G1 & G2 & G3 & G4 & G5 & G6 & G7 & G8 & G9 & G10 \\
\hline G1 & $\mathbf{9 4}$ & 0 & 0 & 0 & 0 & 0 & 0 & 0 & 0 & 0 \\
\hline G2 & 1 & $\mathbf{9 2}$ & 0 & 0 & 0 & 0 & 0 & 0 & 0 & 0 \\
\hline G3 & 0 & 0 & $\mathbf{1 0 0}$ & 0 & 0 & 0 & 0 & 0 & 0 & 0 \\
\hline G4 & 1 & 3 & 0 & $\mathbf{8 4}$ & 0 & 0 & 0 & 0 & 0 & 0 \\
\hline G5 & 0 & 1 & 0 & 0 & $\mathbf{1 0 0}$ & 0 & 0 & 0 & 0 & 0 \\
\hline G6 & 0 & 0 & 0 & 0 & 0 & $\mathbf{1 0 0}$ & 0 & 0 & 0 & 0 \\
\hline G7 & 0 & 1 & 0 & 1 & 0 & 0 & $\mathbf{8 4}$ & 0 & 0 & 0 \\
\hline G8 & 0 & 1 & 0 & 2 & 0 & 0 & 0 & $\mathbf{1 0 0}$ & 0 & 0 \\
\hline G9 & 0 & 0 & 0 & 0 & 0 & 0 & 0 & 0 & $\mathbf{9 6}$ & 0 \\
\hline G10 & 0 & 0 & 0 & 0 & 0 & 0 & 0 & 0 & 0 & $\mathbf{1 0 0}$ \\
\hline \multicolumn{10}{|c|}{ Overall accuracy 95.4\% } \\
\hline
\end{tabular}

TABLE III. ClasSIFICATION OF PQ EVENTS WITH NOISE USING MD CLASSIFIER

\begin{tabular}{|c|l|c|l|l|l|l|l|c|c|c|}
\hline & G1 & G2 & G3 & G4 & G5 & G6 & G7 & G8 & G9 & G10 \\
\hline G1 & $\mathbf{9 5}$ & 0 & 0 & 4 & 0 & 0 & 1 & 0 & 0 & 0 \\
\hline G2 & 1 & $\mathbf{9 1}$ & 0 & 7 & 0 & 0 & 0 & 1 & 0 & 0 \\
\hline G3 & 1 & 0 & $\mathbf{8 9}$ & 5 & 0 & 1 & 0 & 1 & 3 & 0 \\
\hline G4 & 1 & 3 & 0 & $\mathbf{9 3}$ & 0 & 0 & 2 & 1 & 0 & 0 \\
\hline G5 & 0 & 2 & 0 & 3 & $\mathbf{9 4}$ & 0 & 1 & 0 & 0 & 0 \\
\hline G6 & 0 & 0 & 2 & 2 & 0 & $\mathbf{9 3}$ & 0 & 2 & 1 & 0 \\
\hline G7 & 3 & 2 & 0 & 2 & 0 & 0 & $\mathbf{9 1}$ & 2 & 0 & 0 \\
\hline G8 & 1 & 1 & 0 & 6 & 0 & 1 & 0 & $\mathbf{9 0}$ & 1 & 0 \\
\hline G9 & 0 & 0 & 1 & 1 & 2 & 3 & 1 & 0 & $\mathbf{9 2}$ & 0 \\
\hline G10 & 0 & 3 & 0 & 2 & 0 & 0 & 0 & 0 & 0 & $\mathbf{9 5}$ \\
\hline & \multicolumn{10}{|c|}{ Overall accuracy 92.3\% } \\
\hline
\end{tabular}

\section{A. Comparative Study}

The proposed approach, specially the MD classifier, is compared with LVQ (learning vector quantization) and MED (minimum Euclidean distance) classifiers. LVQ, a supervised version of SOM (self organizing map) is used for solving classification problems in particular. Tables IV and V show the comparative performance among LVQ, MED and MD classifiers considering 3, 4, 7, 9 and 10 groups of PQ events.
The overall accuracy of LVQ, MED and MD is found as $90 \%$, 95\% and $96 \%$ when 3 groups of PQ events are tested, see Table IV. Additionally, increasing the number of groups or classes, the comparative study is conducted and results are shown in Table V. From Table V it is evident that performance of LVQ and MED degrades severely as the number of groups increase whereas MD shows excellent performance with 10 groups of PQ events.

TABLE IV. ClassificATION RESUlTS OF PQ EVENTS USING LVQ, MD AND MED CLASSIFIERS CONSIDERING THREE GROUPS

\begin{tabular}{|c|c|c|c|c|c|c|c|c|c|}
\hline & \multicolumn{3}{|c|}{ LVQ } & \multicolumn{3}{c|}{ MED } & \multicolumn{3}{c|}{ MD } \\
\cline { 2 - 11 } & G1 & G2 & G3 & G1 & G2 & G3 & G1 & G2 & G3 \\
\hline G1 & $\mathbf{9 9}$ & 1 & 0 & $\mathbf{9 3}$ & 4 & 3 & $\mathbf{9 4}$ & 0 & 0 \\
\hline G2 & 13 & $\mathbf{8 3}$ & 4 & 3 & $\mathbf{9 6}$ & 1 & 1 & $\mathbf{9 2}$ & 0 \\
\hline G3 & 10 & 3 & $\mathbf{8 7}$ & 3 & 1 & $\mathbf{9 6}$ & 0 & 0 & $\mathbf{1 0 0}$ \\
\hline \multicolumn{4}{|c|}{$\begin{array}{c}\text { Overall accuracy } \\
=90 \%\end{array}$} & $\begin{array}{c}\text { Overall accuracy } \\
=95 \%\end{array}$ & \multicolumn{3}{c|}{$\begin{array}{c}\text { Overall accuracy } \\
=96 \%\end{array}$} \\
\hline
\end{tabular}

TABLE V. CLASSIFICATION RESUlTS OF PQ EVENTS USING LVQ, MD AND MED CLASSIFIERS CONSIDERING MORE THAN THREE GROUPS

\begin{tabular}{|c|c|c|c|}
\hline \multirow{2}{*}{ No. of Groups } & \multicolumn{3}{|c|}{ Accuracy of classification } \\
\cline { 2 - 4 } & LVQ & MED & MD \\
\hline 4 groups (G1-G4) & $90 \%$ & $95.2 \%$ & $92.6 \%$ \\
\hline 7 groups (G1-G7) & $82 \%$ & $91 \%$ & $93.5 \%$ \\
\hline 9 groups (G1-G9) & $70 \%$ & $85 \%$ & $94.4 \%$ \\
\hline 10 groups (G1-G10) & $61 \%$ & $81 \%$ & $95.4 \%$ \\
\hline
\end{tabular}

\section{B. Validation of Proposed Approach using IEEE 1159.2 Working Group Data}

This Section presents the classification results of sinusoidal waveforms of 15 power quality (PQ) events, which have been taken from an authentic source of IEEE 1159.2 working group data [8], see Fig. 3 for illustration. The proposed technique is validated by achieving $100 \%$ successful classification results for 15 voltage signals denoted as PQ event 1 , PQ event 2 and so on. The classification results are shown in Table VI.

TABLE VI. CLASSIFICATION RESULTS OF 15 PQ EVENTS OF IEEE 1159.2 WORKING GROUP DATA USING PROPOSED APPROACH

\begin{tabular}{|c|c|}
\hline Voltage signals & $\begin{array}{c}\text { Classified Power } \\
\text { Quality Events }\end{array}$ \\
\hline PQ event 1,15 & Sag with harmonics \\
\hline PQ event $2,3,4,5,6,7,8,9,10,11,13$, & Flicker \\
\hline PQ event 12 & Transient \\
\hline
\end{tabular}

(a)

(b) 


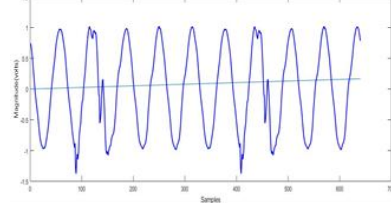

(c)

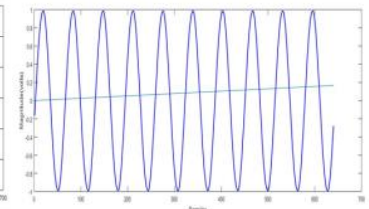

(d)

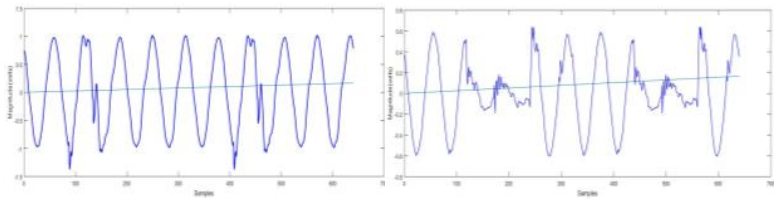

(e)

(f)

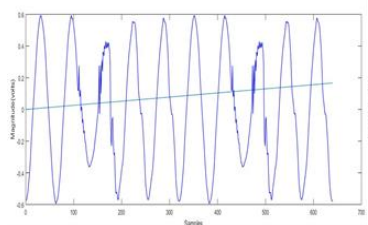

(g)

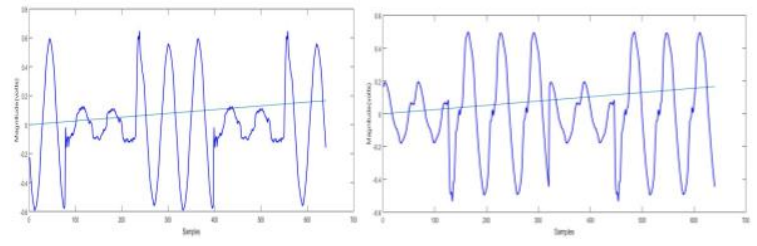

Fig. 3. Voltage Signal affected by deffernet PQ Events [8].

\section{Conclusions}

This paper presents an effective method for detection and classification of power quality (PQ) disturbances or events. The proposed method incorporates $\mathrm{S}$-Transform and Mahalanobis Distance technique; S-Transformation is used to extract four features required for classification and least MD classifier accurately classifies the PQ events. Since proposed approach exploits less number of features, less memory space is needed for classification and it assists to reduce the computational burden to a great range. Furthermore, MD based classification does not require any off-line training of the PQ events; it only requires to form the feature matrix labeled with $P Q$ event-wise groups. Throughout the simulation results it is observed that the proposed method accurately classifies 10 PQ events even under noisy conditions. Hence, it outperforms the wavelet analysis which is prone to noise. Moreover, MD classifier is compared with LVQ and MED classifiers, and the best results are observed from MD based technique. Therefore, the proposed approach would be an effective solution for detection and classification of PQ events.

\section{REFERENCES}

[1] R. C. Dugan and M. F. McGranaghan, Electrical Power Systems Quality, 2nd ed. New York: McGraw-Hill, 2002.

[2] De la Rosa, Francisco, "Harmonics and power systems" CRC press, 2006.

[3] J. Schlabbach, D. Blume and T. Stephanblome, "Voltage Quality in Electrical Power System", Institution of Electrical Engineers, London, 2001.

[4] Fathi, H. M. E. "Power quality assessment." Journal of Al-Azhar University Cairo, Egypt, 2012.
[5] S. Santoso, W. M. Grady, E. J. Powers, J. Lamoree and S. C. Bhatt,"Characterization of distribution power quality events with Fourier and wavelet transforms," in IEEE Transactions on Power Delivery, vol. 15, no. 1, pp. 247-254, Jan 2000.

[6] Ying-Tung Hsiao, Cheng-Long Chuang and Joe-Air Jiang, "Recognition of power quality events using wavelet-based dynamic structural neural networks," in proc. 2005 IEEE International Symposium on Circuits and Systems, pp. 3885-3888, vol. 4, 2005.

[7] M. R. Alam, K. M. Muttaqi and A. Bouzerdoum, "A short length windowbased method for islanding detection in distributed generation," The 2012 International Joint Conference on Neural Networks (IJCNN), Brisbane, QLD, 2012, pp. 1-6.

[8] IEEE 1159.2 working group data, available online: "grouper.ieee.org/group/1159.2/testwave.html", accessed on $2^{\text {nd }}$ august 2017.

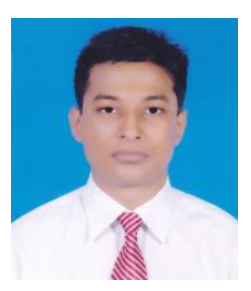

Md Jashim Uddin Bhuiyan received the B.Sc. degree in electrical and electronic engineering (EEE) from American International University-Bangladesh (AIUB), Bangladesh, in august 2014.Currently he is a student of MSc in EEE at AIUB. Now he is working at British American Tobacco Bangladesh (BATB) under supply chain management. His research interests mainly include fault detection in power system, application of artificial intelligence in power

quality events classification.

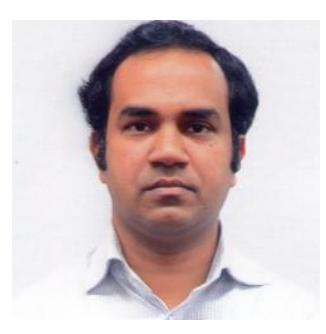

Mollah Rezaul Alam received the B.Sc degree in electrical and electronic engineering from Bangladesh University of Engineering and Technology (BUET), Bangladesh in 2005, and the Ph.D. degree in Electrical and Electronic Engineering from University of Wollongong, New South Wales, Australia in 2015. Currently, he is an Assistant Professor in the EEE department of American International University-

Bangladesh (AIUB). Prior to completing Ph.D. degree, he was involved in the telecommunication industry in Bangladesh for 5 years, where he worked in the area of Intelligent Network \& Value Added Services of cellular mobile technology. His research interests include computational intelligence, data mining, fault detection, classification and analysis considering the impacts of distributed energy resources. 